\title{
Intraoperative Photoactivated Chromophore for Infectious Keratitis-Corneal Cross-Linking (PACK- CXL) During Penetrating Keratoplasty for the Management of Fungal Keratitis in an Immunocompromised Patient
}

\author{
Dimitrios G. Mikropoulos • George D. Kymionis • Nafsika Voulgari • Eirini Kaisari • \\ Konstantinos A. Nikolakopoulos • Andreas Katsanos · Anastasios G. Konstas
}

Received: May 24, 2019 / Published online: July 5, 2019

(C) The Author(s) 2019

\section{ABSTRACT}

Introduction: To present a novel intraoperative application of photoactivated chromophore for infectious keratitis-corneal cross-linking (PACK$\mathrm{CXL}$ ) in the management of post-penetrating keratoplasty (PKP) multiresistant fungal keratitis in a patient with irradiation-related local immunosuppression.

Case report: A 62-year-old female underwent uneventful PKP for the management of post-

Enhanced digital features To view enhanced digital features for this article go to https://doi.org/10.6084/ m9.figshare.8320256.

D. G. Mikropoulos

3rd University Department of Ophthalmology,

Aristotle University of Thessaloniki, Thessaloniki, Greece

G. D. Kymionis · N. Voulgari - E. Kaisari ·

K. A. Nikolakopoulos

Ophthalmology Department, Jules Gonin Eye

Hospital, University of Lausanne, Lausanne,

Switzerland

\section{A. Katsanos}

Department of Ophthalmology, University of

Ioannina, Ioannina, Greece

A. G. Konstas $(\bowtie)$

1st and 3rd University, Department of

Ophthalmology, Aristotle University of

Thessaloniki, Thessaloniki, Greece

e-mail: konstas@med.auth.gr irradiation actinic keratopathy. Three months postoperatively, she presented with a diffuse corneal melting abscess that was infiltrating the donor-recipient junction. Despite intensive antibiotic and antifungal therapy, corneal melting progressed to graft perforation. A repeat PKP combined with intraoperative PACK-CXL was performed. PACK-CXL was applied initially on the infected graft, involving the corneoscleral rim and then following placement of the donor button. No intra- or postoperative graftrelated complications were encountered. No signs of infection were noted, and the graft remained clear during the 9-month follow-up period.

Conclusion: Intraoperative PACK-CXL combined with PKP appears to be a safe and effective technique for the management of post-PKP resistant fungal keratitis.

Keywords: Corneal cross-linking; Fungal keratitis; PACK-CXL; Penetrating keratoplasty

\section{INTRODUCTION}

Infectious keratitis constitutes a devastating complication of penetrating keratoplasty (PKP) with a reported incidence ranging from 1.76 to 4.9\%. Fungal keratitis incidence has increased considerably from 9.8 to $66.7 \%$ of infectious keratitis following PKP, accounting for $4.3 \%$ of PKPs overall [1]. Typically, post-PKP fungal 
keratitis is managed with intensive topical and systemic antifungal therapy including intrastromal or intracameral antifungal injections [1]. Nevertheless, graft viability is often put at risk resulting in graft failure necessitating a repeat keratoplasty or even evisceration in the case of intractable intraocular spreading of the infection. Corneal cross-linking (CXL) has been recently introduced in the management of infectious keratitis with an increasing number of publications verifying its successful microbicidal properties [2, 3]. Nevertheless, studies on the efficacy of the photoactivated chromophore for infectious keratitis-corneal cross-linking (PACK-CXL) in the therapeutic profile of fungal corneal infections remain somewhat inconclusive [4-7]. Herein, we present a novel intraoperative application of PACK-CXL in the management of post-PKP extensive resistant fungal keratitis in a patient with irradiation-induced local immunosuppression.

\section{CASE REPORT}

Written informed consent for the publication of this article was obtained from the patient prior to submission of the manuscript.

A 62-year-old female with a past ocular history of conjunctival melanoma in the left eye, which had been treated with topical excision and beta brachytherapy with ruthenium 10 years earlier, was referred to our department for the management of actinic keratopathy. Uncorrected distance visual acuity (UCVA) at presentation was Counting Fingers (CF) at $50 \mathrm{~cm}$. The patient underwent uneventful penetrating keratoplasty, and the 1-month postoperative UCVA was CF at $1 \mathrm{~m}$. Three months postoperatively she presented as an emergency to our department complaining of red painful eye and reduced visual acuity. UCVA had decreased to hand movement (HM). Slit-lamp examination revealed conjunctival injection and a diffuse corneal melting abscess infiltrating the corneal rim (Fig. 1a). Corneal scraping was performed and sent for microbiologic cultures and analysis. The patient reported professional exposure to plant material, making a fungal infection highly possible. Intensive antibiotic and antifungal therapy was introduced including voriconazole topical and per os, amphotericin $\mathrm{B}$ per os, topical moxifloxacine and doxycycline. Cultures confirmed fungal keratitis with Purpureocillium lilacinum (anc. Paecilomyces lilacinus), which was voriconazolesensitive, and this treatment was subsequently pursued. Despite aggressive medical treatment, corneal melting progressed to the stage of graft perforation. We decided to proceed with a combined repeat PKP and PACK-CXL. The operation was performed under general anesthesia without any intraoperative complication. PACK-CXL was applied intraoperatively on the infected graft. A modified protocol was utilized with riboflavin instillation every $2 \mathrm{~min}$ for $15 \mathrm{~min}$ followed by UVA irradiation at $9 \mathrm{~mW}$ for $10 \mathrm{~min}$. The irradiation zone was set from limbus to limbus to cover an area extending over the donor-recipient junction and the corneoscleral rim, as it seemed that the infection had spread to the recipient rim. PACK-CXL was repeated after placement of the new graft with the same irradiation zone and according to the same parameters for $10 \mathrm{~min}$. Immediate postoperative UCVA was $\mathrm{CF}$ at $30 \mathrm{~cm}$. The postoperative period was uneventful, and the graft remained clear thereafter. Nine months postoperatively the patient had regained a UCVA of $\mathrm{CF}$ at $1 \mathrm{~m}$. No signs of infection or graft-related complications were noted (Fig. 1b).

\section{DISCUSSION}

To the best of our knowledge, this is the first report of intraoperative PACK-CXL application combined with penetrating keratoplasty for the management of refractory fungal keratitis on a corneal graft specifically in a patient with irradiation-related local immunosuppression. Control of the infection was achieved without complications; graft viability was not jeopardized, and visual acuity improved to the level of pre-infection vision.

A growing body of evidence supports the role of CXL in the treatment of infectious keratitis $[2,3]$. Its microbicidal properties make PACK- 


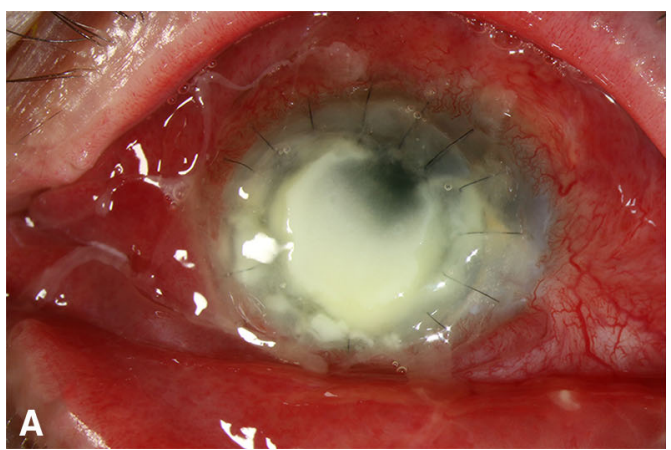

Fig. 1 Slit-lamp photo a Three months post-penetrating keratoplasty showing a diffuse corneal melting abscess infiltrating the corneoscleral rim and b 6 months after combined corneal cross-linking and penetrating

CXL a promising alternative to conventional antimicrobial therapy in the management of multi-drug-resistant corneal infections. Experimental and clinical studies have demonstrated the antimicrobial efficacy of CXL in vitro and in vivo in refractory infectious keratitis, with higher efficiency in the early period after diagnosis. It is worth noting that PACK-CXL can be employed as an adjunct treatment modality supplementary to antimicrobial medication [5] as well as first-line therapy [8]. In addition to its pathogen-eradicating properties, the synergistic effect of UV-A light and riboflavin increases tissue resistance to enzymatic degradation, thus halting the corneal melting process. In the setting of penetrating keratoplasty, theoretically, it enhances graft-recipient adhesion, while diminishing the risk of graft rejection.

Nevertheless, evidence remains controversial regarding the antifungal effect of PACK-CXL. Kashiwabuchi et al. [4] reported no visible efficacy in their in vitro study. In contrast, Zhu et al. [7] showed a significant effect against Fusarium solani both in vitro and in vivo. Fungal infections typically present with deep invasion of the corneal stroma, usually disseminating to the anterior chamber, thus limiting the efficacy of PACK-CXL and risking endothelial cell damage with subsequent corneal edema. Uddaraju et al. [6] reported no efficacy of PACKCXL as adjuvant to standard medical treatment in recalcitrant deep stromal fungal ulcers and risk of perforation. This observation was also

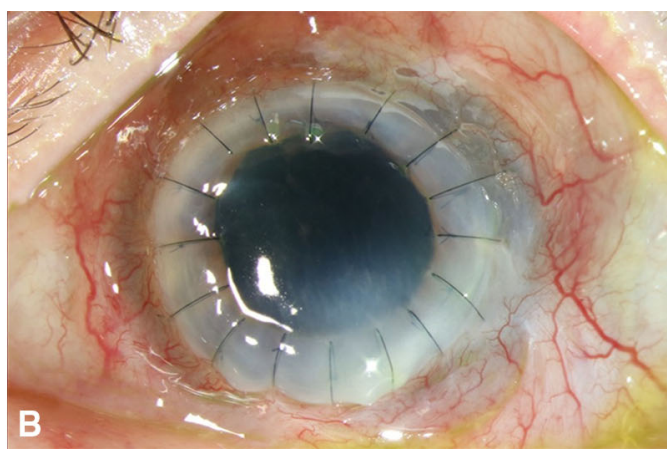

keratoplasty, showing complete resolution of infection and a clear graft that remained stable thereafter

supported by a more recent study from Kasetsuwan et al. [5]. Therefore, the therapeutic effect of PACK-CXL appears greater in early fungal corneal infections before deeper progression occurs. In our case, cultures revealed the presence of Purpureocillium lilacinum (Paecilomyces sp.), a filamentous fungus that comprises an extremely rare cause of fungal keratitis [1]. Moreover, due to the previous history of ocular irradiation, the eye of our case was immunocompromised, which could have contributed to the inadequate therapeutic response to the topical and systemic antifungal treatment employed.

The extensive stromal infection of the corneal graft and the subsequent significant corneal melting and graft perforation which occurred in our immunocompromised patient made a repeat PKP inevitable. Therapeutic keratoplasty aims to decrease the infection load and preserve globe integrity and may effectively treat infectious keratitis along with medical therapy [9]. In our case, it was combined with PACK-CXL, which was performed with an irradiation zone adapted to cover the corneoscleral rim to eradicate any residual infection. It has been shown that application of CXL on the limbus does not affect the regenerative capacity of limbal epithelial cells [10]. A previous report [11] has demonstrated the successful management of post-PKP resistant MRSA keratitis with PACK-CXL performed on the graft. Conversely to ours in that case, the abscess was limited to 
the 3-5 o'clock area and was not associated with corneal melting and graft failure. In a recent case report, Balparda et al. [12] proposed the application of PACK-CXL combined with PKP for the treatment of severe marginal Fusarium spp. keratitis on a virgin cornea. Due to the corneal perforation in that case, the authors performed PKP followed by decentered PACKCXL on the graft to eradicate the residual peripheral fungal elements.

\section{CONCLUSION}

We report a case where intraoperative PACKCXL application was combined successfully with penetrating keratoplasty in the management of refractory fungal keratitis in a patient with irradiation-related local immunosuppression. This approach led to elimination of the infection without complications and graft viability. Fungal infections after keratoplasty represent a significant challenge to the corneal surgeon. Our case highlights the fact that in these testing circumstances the application of PACK-CXL appears to be a safe and potentially effective approach.

\section{ACKNOWLEDGEMENTS}

Funding. No funding or sponsorship was received for this study or the publication of this article.

Authorship. All named authors meet the International Committee of Medical Journal Editors (ICMJE) criteria for authorship for this article, take responsibility for the integrity of the work as a whole, and have given their approval for this version to be published.

Disclosures. Dimitrios G. Mikropoulos, George D. Kymionis, Nafsika Voulgari, Eirini Kaisari, Konstantinos A. Nikolakopoulos and Andreas Katsanos have nothing to disclose. Anastasios G. Konstas is a member of the journal's Editorial Board.
Compliance with ethics guidelines. Written informed consent for the publication of this report was obtained from the patient.

Open Access. This article is distributed under the terms of the Creative Commons Attribution-NonCommercial 4.0 International License (http://creativecommons.org/licenses/ by-nc/4.0/), which permits any noncommercial use, distribution, and reproduction in any medium, provided you give appropriate credit to the original author(s) and the source, provide a link to the Creative Commons license, and indicate if changes were made.

\section{REFERENCES}

1. Lin I-H, Chang Y-S, Tseng S-H, Huang Y-H. A comparative, retrospective, observational study of the clinical and microbiological profiles of postpenetrating keratoplasty keratitis. Sci Rep. 2016;6(1):32751. https://doi.org/10.1038/srep32751.

2. Papaioannou L, Miligkos M, Papathanassiou M. Corneal collagen cross-linking for infectious keratitis: a systematic review and meta-analysis. Cornea. 2016;35(1):62-71. https://doi.org/10.1097/ico. 0000000000000644 .

3. Tabibian D, Mazzotta C, Hafezi F. PACK-CXL: corneal cross-linking in infectious keratitis. Eye Vis. 2016;3(1):11. https://doi.org/10.1186/s40662-0160042-x.

4. Kashiwabuchi RT, Carvalho FRS, Khan YA, Hirai F, Campos MS, McDonnell PJ. Assessment of fungal viability after long-wave ultraviolet light irradiation combined with riboflavin administration. Graefe Arch Clin Exp Ophthalmol. 2013;251(2):521-7. https://doi.org/10.1007/s00417-012-2209-z.

5. Kasetsuwan N, Reinprayoon U, Satitpitakul V. Photoactivated chromophore for moderate to severe infectious keratitis as an adjunct therapy: a randomized controlled trial. Am J Ophthalmol. 2016;165:94-9. https://doi.org/10.1016/j.ajo.2016. 02.030 .

6. Uddaraju M, Mascarenhas J, Das MR, et al. Corneal cross-linking as an adjuvant therapy in the management of recalcitrant deep stromal fungal keratitis: a randomized trial. Am J Ophthalmol. 2015;160(1):131-1345.e5. https://doi.org/10.1016/ j.ajo.2015.03.024. 
7. Zhu Z, Zhang H, Yue J, Liu S, Li Z, Wang L. Antimicrobial efficacy of corneal cross-linking in vitro and in vivo for Fusarium solani: a potential new treatment for fungal keratitis. BMC Ophthalmol. 2018;18(1):65. https://doi.org/10.1186/ s12886-018-0727-0.

8. Makdoumi K, Mortensen J, Sorkhabi O, Malmvall B-E, Crafoord S. UVA-riboflavin photochemical therapy of bacterial keratitis: a pilot study. Graefe's Arch Clin Exp Ophthalmol. 2012;250(1):95-102. https://doi.org/10.1007/s00417-011-1754-1.

9. Raj A, Bahadur H, Dhasmana R. Outcome of therapeutic penetrating keratoplasty in advanced infectious keratitis. J Curr Ophthalmol. 2018;30(4):315-20. https://doi.org/10.1016/j.joco.2018.04.001.

10. Richoz O, Tabibian D, Hammer A, Majo F, Nicolas M, Hafezi F. The effect of standard and high-fluence corneal cross-linking (CXL) on cornea and limbus. Invest Ophthalmol Vis Sci. 2014;55(9):5783-7. https://doi.org/10.1167/iovs.14-14695.

11. Labiris G, Giarmoukakis A, Larin R, Sideroudi H, Kozobolis VP. Corneal collagen cross-linking in a late-onset graft infectious ulcer: a case report. J Med Case Rep. 2014;8(1):180. https://doi.org/10.1186/ 1752-1947-8-180.

12. Balparda K, Mejia-Turizo JC, Herrera-Chalarca T. Simultaneous noncentered photoactivated chromophore for keratitis-corneal collagen cross-linking and penetrating keratoplasty for treatment of severe Marginal Fusarium spp. keratitis: a description of a new surgical technique. Case Rep Ophthalmol Med. 2017;2017:6987896. https://doi.org/10.1155/2017/ 6987896 . 